\title{
Cylindrical or T-shaped Silicone Rubber Stents for Microanastomosis
}

\author{
-Technical Note-
}

\author{
Yoshikazu OKADA, Takeshi SHIMA*, Kanji YAMANE*, Chie YAMANAKA*, \\ and Reiko KAGAWA
}

\author{
Department of Neurosurgery, Shimane Medical University, Izumo, Shimane; *Department \\ of Neurosurgery, Chugoku Rousai Hospital, Kure, Hiroshima
}

\begin{abstract}
The ostium of the recipient artery and the orifice of the donor artery must be clearly visualized for the establishment of microvascular anastomosis. Specially designed colored flexible cylindrical or Tshaped silicone rubber stents were made in various sizes (400 or $500 \mu \mathrm{m}$ diameter and $5 \mathrm{~mm}$ length) and applied to bypass surgery in patients with occlusive cerebrovascular disease such as moyamoya disease and internal carotid artery occlusion. The colored flexible stents facilitated confirmation of the ostium of the artery even in patients with moyamoya disease and allowed precise microvascular anastomosis without problems caused by the stent.
\end{abstract}

Key words: silicone rubber stent, superficial temporal artery-middle cerebral artery anastomosis, extracranial-intracranial anastomosis

\section{Introduction}

The extracranial-intracranial (EC-IC) bypass trial has concluded that surgery provides no overall benefits against stroke from the surgery. ${ }^{11}$ However, the superficial temporal artery-middle cerebral artery (STA-MCA) anastomosis may be effective in adults with moyamoya disease causing ischemic symptoms. ${ }^{2,4,8)}$ Recent work has suggested that by pass surgery may also be effective in patients with hemodynamic compromise due to occlusive cerebrovascular disease. ${ }^{5)}$ The major requirements for effective EC-IC bypass are complete accomplishment of the surgical preparations without complications and steady patency with an appropriate bypass flow. Surgical preparations for EC-IC bypass surgery are more difficult in patients with very small, thin, fragile, and transparent recipient arteries. The donor artery may also cause problems due to intimal dissection associated with atherosclerotic changes. The application of a stent for microanastomosis is a well-known method, but no suitable stent is available for bypass surgery. ${ }^{6}{ }^{6} \mathrm{We}$

Received June 1, 1998;

Accepted September 2, 1998 have developed cylindrical or T-shaped colored silicone rubber stents for reliable performance of EC-IC bypass surgery.

\section{Materials and Methods}

The cylinder stents were made by catalytic polymerization of silicone rubber (Xythantopren L; Bayer Dental, Leverkusen, Germany) in molds made of $5 \mathrm{~mm}$ long polyethylene tubes (Intravenous Cannula Set; Portex, Kent, U.K.). We mixed 7-10 ml of silicone rubber with a drop of catalyst. The silicone cylinders (approximately $400 \mu \mathrm{m}$ and $500 \mu \mathrm{m}$ in diameter and $5 \mathrm{~mm}$ in length) were extruded by simple pinch pressure applied to the end of the mold (Fig. 1 left). The T-shaped stents were made by vertically suturing one cylindrical stent to the mid point of another with 10-0 nylon monofilament (Fig. 1 right).

STA-MCA anastomosis was performed in 30 patients with moyamoya disease and 50 patients with internal carotid artery (ICA) occlusion. In addition, vein graft bypass was performed in 22 patients.

Figure 2 shows the STA-MCA anastomosis in a 


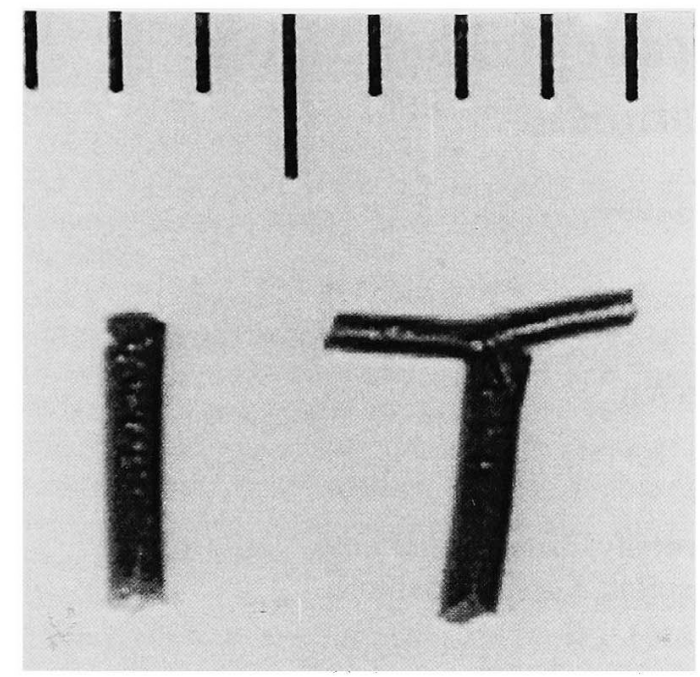

Fig. 1 left: Blue colored cylindrical silicone rubber stent $(500 \mu \mathrm{m}$ ouside diameter [OD]). right: $\mathrm{T}$-shaped silicone rubber stent $(400$ $\mu \mathrm{m}$ OD horizontal stent and $500 \mu \mathrm{m}$ OD vertical stent).

patient with moyamoya disease using our cylindrical stent. The small recipient cortical artery was arteriotomized to about $2.0 \mathrm{~mm}$ in length. Then a blue-colored cylindrical stent ( $400 \mu \mathrm{m}$ in diameter) was settled in the arteriotomized artery with adjustment of its length and size. The stent was flexible enough to be inserted into the arterial lumen without causing any intimal injury. And the stent could be easily removed through the small residual orifice for the final three sutures without damaging the arterial walls. The stent clearly visualized the ostium of the arteriotomy and facilitated the suturing the recipient MCA and the donor STA.

Postoperative angiography revealed excellent patency of the anastomosis in all patients with moyamoya disease, 49 of 50 patients with ICA occlusion, and 21 of 22 patients with vein graft bypass.

\section{Discussion}

Our silicone rubber stents enabled successful microvascular anastomosis even in patients with moyamoya disease, which is associated with extremely small, fragile, and transparent cortical arteries. Successful performance of microvascular anastomosis requires clear visualization of the ostium of the recipient artery and the orifice of the donor artery. The former is more important in microvascular anastomosis for moyamoya disease. Our $400 \mu \mathrm{m}$ colored silicone rubber stent is so flexible that placement in the recipient artery is very easy
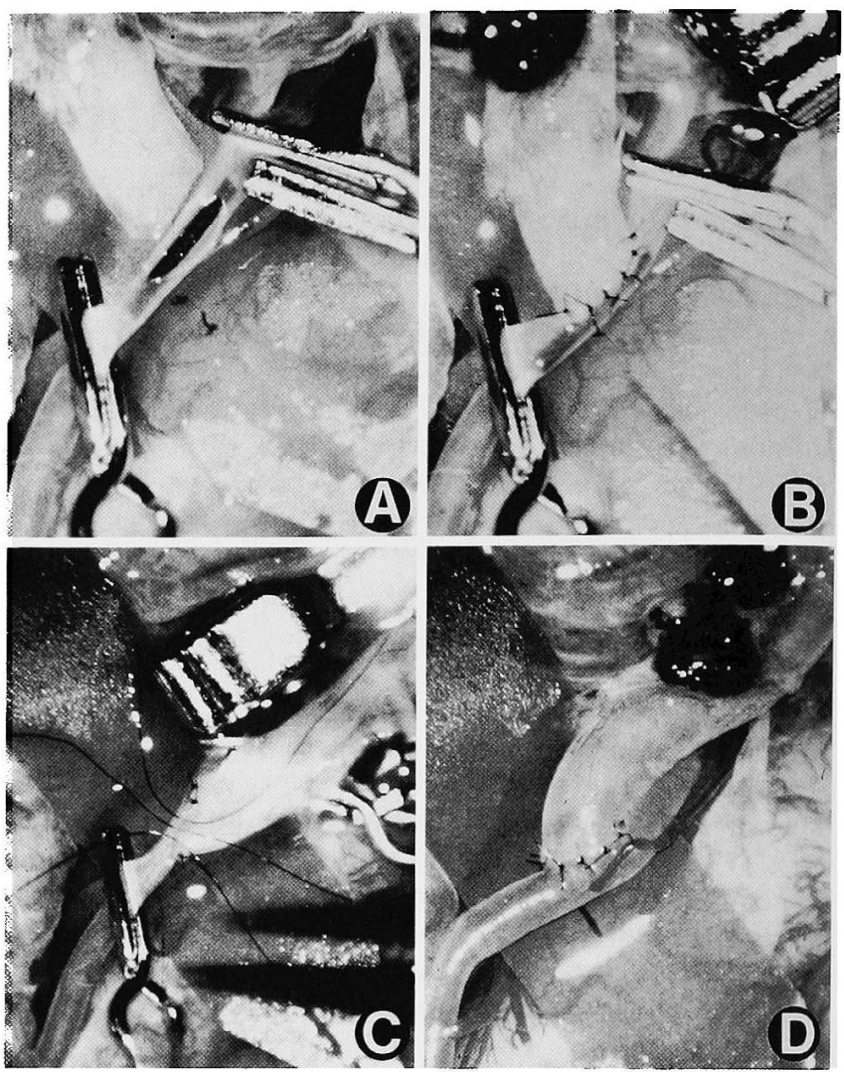

Fig. 2 Operative photographs showing the procedures for superficial temporal artery-middle cerebral artery anastomosis using a silicone stent in a patient with moyamoya disease. A: The recipient cortical artery is arteriotomized and the stent (blue silicone cylinder, $400 \mu \mathrm{m}$ outside diameter) is inserted into the recipient artery. The ostium of the recipient artery can be clearly seen. B: One side of the anastomosis is completed, where the residual recipient and donor arterial wall can be easily observed. C: The final three sutures are performed without ties and the stent is removed. D: After completion of the anastomosis, excellent blood flow through the bypass is observed.

and non-traumatic to the intima. Furthermore, the blue color of the stent allows easy visualization of the thin and transparent cortical arterial wall. The latter is important in patients with dissection of the intima of the donor artery, which would prevent suturing of the whole arterial wall and cause occlusion of the anastomosis. Additionally the discrepancy of the size between the recipient cortical artery and the donor vein in vein graft bypass requires many meticulous sutures and may involve the contralateral wall of the graft vein. ${ }^{7)}$ These problems 
can be resolved by separating the bilateral donor wall with our $\mathrm{T}$-shaped silicone rubber stent.

An interesting visualization method for arteriotomy based on blue staining with methylrosaniline chloride (pyoctanium blue) has been used in bypass surgery for children with moyamoya disease. ${ }^{\text {3) }}$ The process enabled the surgeons to create anastomoses precisely and quickly with an excellent patency rate, but the safety of the dye remains untested. The application of a stent for microanastomosis is not a new idea, ${ }^{6}$ but the hardness of the conventional stent is the most serious problem because a hard stent may damage the intima of the recipient artery. Our stent was made of silicone rubber, which is widely used by dentists without problems, and had no harmful effects in our bypass series. The optimum hardness can be obtained in our stents by adjusting the volume of catalyst. Therefore, suitable stents can be easily made by our method.

\section{References}

1) The EC/IC Bypass Group: Failure of extracranial/intracranial arterial bypass to reduce the risk of ischemic stroke. Results of an international randomized trial. N Engl J Med 313: 1191-1200, 1985

2) Houkin $K$, Kamiyama H, Abe H, Takahashi A, Kuroda S: Surgical therapy for adult moyamoya disease. Can surgical revascularization prevent the recurrence of intracerebral hemorrhage? Stroke 27: 1342-1346, 1996

3) Kamiyama H, Takahashi A, Houkin K, Mabuchi S, Abe $\mathrm{H}$ : Visualization of the ostium of an arteriotomy in bypass surgery. Neurosurgery 33: 1109-1110, 1993

4) Karasawa J, Kikuchi H, Furuse S, Kawamura J, Sakaki T: Treatment of moyamoya disease with STA-MCA anastomosis. J Neurosurg 49: 679-688, 1978

5) Klijn CJM, Kappelle LJ, Tulleken CAF, van Gijn J: Symptomatic carotid artery occlusion. A reappraisal of hemodynamic factors. Stroke 28: 2084-2093, 1997

6) Ojemann RG, Crowell RM: Surgical Management of Cerebrovascular Disease. Baltimore, Williams \& Wilkins, 1983, pp 71-92

7) Okada $Y$, Shima $T$, Nishida $M$, Yamane $K$ : Retroauricular subcutaneous Dacron tunnel for extracranial-intracranial autologous vein bypass graft. J Neurosurg 81: 800-802, 1994

8) Okada $Y$, Shima T, Nishida M, Yamane K, Yamada T, Yamanaka C: Effectiveness of superficial temporal artery-middle cerebral artery anastomosis in adult moyamoya disease. Cerebral hemodynamics and clinical course in ischemic and hemorrhagic varieties. Stroke 29: 625-630, 1998

Address reprint requests to: Y. Okada, M.D., Department of Neurosurgery, Shimane Medical University, 89-1
Enya-cho, Izumo, Shimane 693-8501, Japan.

\section{Commentary}

The authors have developed cylindrical or T-shaped colored silicone rubber stents and emphasize the usefulness of this stent for microanastomosis in patients with moyamoya disease. We know that the STA-MCA anastomosis of moyamoya vessels is sometimes troublesome because of the thin and transparent cortical artery or the discrepancy in size between the recipient and donor arteries. Especially, clear identification of the ostium of the recipient arterial wall holds the key for successful results. A couple of ideas to use intraluminal stent have been proposed as a simple and reasonable procedure, but no methods has been selected as the optimum. For the skilled surgeon, these methods may be unnecessary and sometimes too complicated. However, it is incumbent on surgeons to make every effort to accomplish perfect anastomosis with more excellent results than before. I am strongly interested in the proposed system and commend the authors for this work.

Shunro ENDO, M.D.

Department of Neurosurgery

Toyama Medical and Pharmaceutical University Toyama, Japan

Based on extensive experience with STA-MCA anastomosis for moyamoya disease, the authors have designed and manufactured a silicone rubber stent. As the authors stated, the use of a stent is not a new idea. Even a soluble stent was experimentally developed in combination with non-suture anastomosis. ${ }^{1)} 1$ personally had a limited experience of using a 3-0 nylon suture and a small silicone tube as a stent. In brief, I found them not very practical since the former was too hard and the latter was not colored. Since the stent of the authors is colored, flexible and 0.4-0.5 $\mathrm{mm}$ in diameter, it will be very helpful in clearly visualizing the ostium of the thin-walled recipient artery and facilitate anastomotic procedures. I congratulate them on this idea and their success in manufacturing the stent using silicone rubber and polyethylene tube. The authors stated in the discussion that their T-shaped silicone rubber stent is useful for vein graft bypass, but this does not seem to be very convincing to me. I believe that practical use is one of the most important issues for these items to be widely used in this field.

In this paper the authors have surely tempted the readers to try the stent at the first opportunity. I hope that such a stent will become more accessible, i.e. commercially available, in the near future. 


\section{Reference}

1) Yamagata S, Carter LP, Handa H, Yonekawa Y, Taki W, Ikada Y, Iwata H: Experimental studies in nonsuture end-to-side microvascular anastomosis. Neurol Med Chir (Tokyo) 21: 701-708, 1981

Shiro NaGaSawa, M.D. Department of Neurosurgery Osaka Medical College Osaka, Japan

Okada et al. have described the use of a silicone stent to facilitate the anastomosis of small vessels for EC-IC bypass. The stent is helpful because of its soft and pliable nature and also that it holds the edges of the arteriotomy apart preventing crucial errors in the anastomosis with such thin walled vessels. It could be made in a variety of sizes to suit different vessels. The coloring of the silicone is important to render the almost transparent vessel wall visible. Their excellent bypass patency rates substantiate the usefulness of the stent. I believe this is an important adjunct and should be considered in the anastomosis of such small vessels.

Chandranath SEN, M.D. Department of Neurosurgery The Mount Sinai Medical Center New York, U.S.A. 